Lauro Augusto Veloso Costa

\title{
ANÁLISE DE 500 RECONSTRUÇÕES DO LIGAMENTO CRUZADO ANTERIOR DE UM REGISTRO INSTITUCIONAL PRIVADO
} Dissertação apresentada à Sociedade
Beneficente Israelita Brasileira Albert
Einstein para obtenção do título de Mestre
em Ciências da Saúde.

São Paulo 


\title{
ANÁLISE DE 500 RECONSTRUÇÕES DO LIGAMENTO CRUZADO ANTERIOR DE UM REGISTRO INSTITUCIONAL PRIVADO
}

\author{
Dissertação apresentada à Sociedade \\ Beneficente Israelita Brasileira Albert \\ Einstein para obtenção do título de Mestre \\ em Ciências da Saúde.
}

Orientador: Prof. Dr. Mário Ferretti Coorientadora: Prof ${ }^{a}$. Eliane Antonioli 
Costa, Lauro Augusto Veloso

Análise de $\mathbf{5 0 0}$ reconstruções do ligamento cruzado anterior de um registro institucional privado / Lauro Augusto Veloso Costa. -- São Paulo, 2018. $\mathrm{x}, 35 \mathrm{f}$.

Dissertação (Mestrado) - Sociedade Beneficente Israelita Brasileira Albert Einstein. Instituto Israelita de Ensino e Pesquisa Albert Einstein. Programa de Pós-Graduação em Ciências da Saúde.

Título em inglês: Analysis of 500 anterior cruciate ligament reconstructions from a private institutional register.

1. Reconstrução do ligamento cruzado anterior. 2. Procedimentos ortopédicos. 3. Doenças musculoesqueléticas/cirurgia

NLM - WE 870

Elaborada pelo Sistema Einstein Integrado de Bibliotecas 
SOCIEDADE BENEFICENTE ISRAELITA BRASILEIRA ALBERT EINSTEIN

Coordenador do Curso de Pós-Graduação: Prof. Dr. Luiz Vicente Rizzo 


\section{Lauro Augusto Veloso Costa}

\section{ANÁLISE DE 500 RECONSTRUÇÕES DO LIGAMENTO CRUZADO ANTERIOR DE UM REGISTRO INSTITUCIONAL PRIVADO}

Presidente da banca:

Prof. Dr. Mário Ferretti

BANCA EXAMINADORA

Membros titulares:

Prof. Dr. Reynaldo Jesus Garcia Filho

Prof. Dr. Luciano Miller Reis Rodrigues

Prof. Dr. Rodrigo Pereira Guimarães

Membros suplentes:

Prof. Dr. Alberto Ofenhejm Gotfryd

Prof. Dr. Pedro Henrique Isoldi Pohl

Aprovada em: 26/04/2018 


\section{Dedicatória}

A Deus, pois sem Ele, não teria forças para prosseguir com meus objetivos.

Aos meus pais, Lauro e Mônica, pelo apoio incondicional durante toda a minha vida até aqui. Graças ao esforço deles tudo isso está sendo possível.

À minha esposa, Natasha, por me apoiar em tudo o que faço. E pelo companheirismo e conselhos valiosos que me permitem caminhar com mais segurança.

Ao meu orientador, Mário, por ter confiado em mim e me cedido essa grande oportunidade. Serei eternamente grato.

À minha coorientadora, Eliane, pelas contribuições intelectuais durante o desenvolvimento dessa dissertação.

Ao meu amigo, Noel, pela contribuição na pesquisa e pelas boas conversas que ajudaram a facilitar essa difícil jornada. 


\section{Agradecimentos}

Ao serviço de estatística do Instituto de Ensino e Pesquisa do Hospital Israelita Albert Einstein.

Aos membros do grupo de pesquisa do Departamento do Aparelho Locomotor do Hospital Israelita Albert Einstein. 


\section{Sumário}

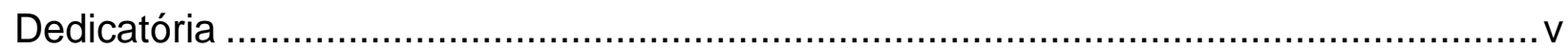

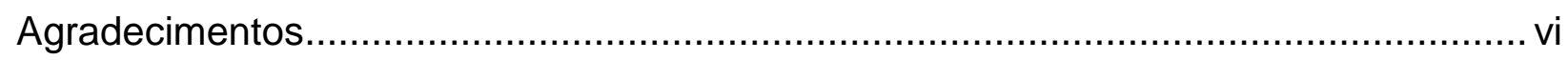

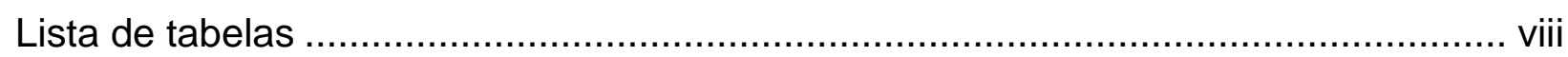

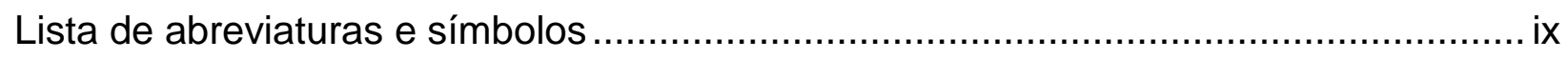

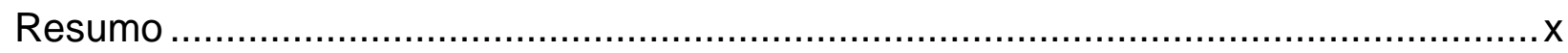

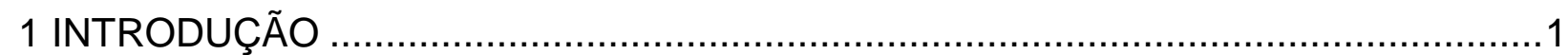

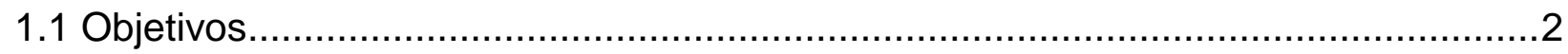

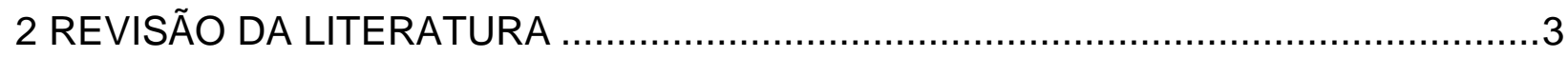

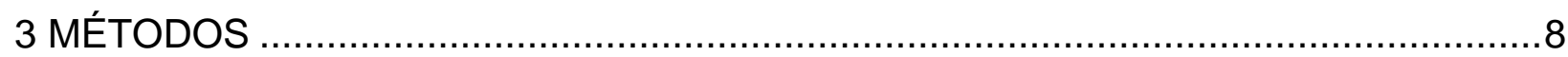

3.1 Registro institucional do ligamento cruzado anterior ..................................... 8

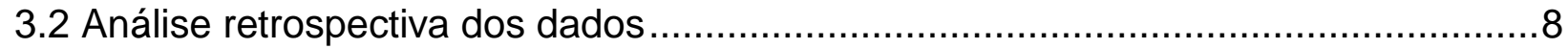

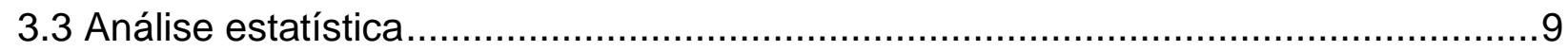

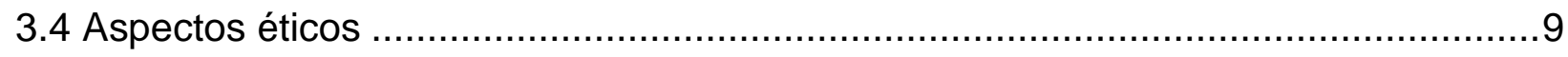

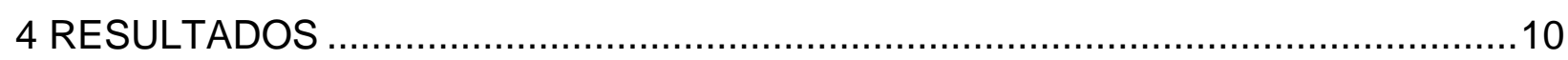

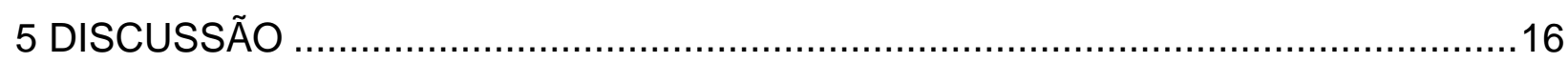

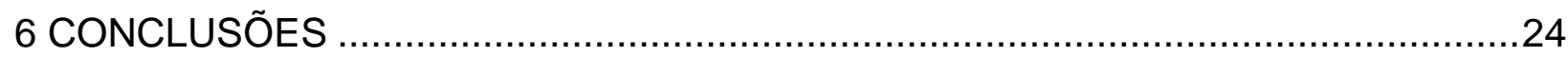

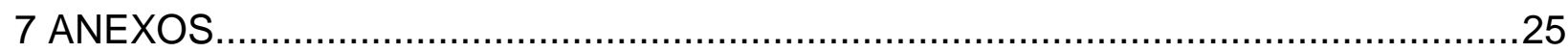

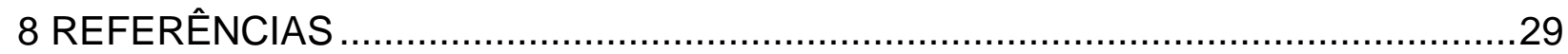

Abstract 


\section{Lista de tabelas}

Tabela 1. Dados demográficos da amostra................................................... 10

Tabela 2. Informações intraoperatórias....................................................... 12

Tabela 3. Informações sobre lesão meniscal e tipo de tratamento .............................13

Tabela 4. Informações do período pós-operatório imediato ....................................14

Tabela 5. Associação entre lesão meniscal e as seguintes variáveis: sexo, idade, índice de massa corporal e tempo de lesão.............................................................. 15 


\section{Lista de abreviaturas e símbolos}

IMC Índice de massa corporal

LCA Ligamento cruzado anterior

LP Ligamento patelar

RLCA Reconstrução do ligamento cruzado anterior

TF Tendões flexores

TVP Trombose venosa profunda 


\section{Resumo}

Introduction: A lesão do ligamento cruzado anterior é uma lesão comum, especialmente em atletas, além de ser também a lesão ligamentar do joelho que mais frequentemente necessita de reconstrução cirúrgica. Objetivos: Descrever as características epidemiológicas de pacientes submetidos à reconstrução do ligamento cruzado anterior, bem como as características da prática médica desse procedimento. Métodos: Foram revisados retrospectivamente os dados dos pacientes submetidos à reconstrução do ligamento cruzado anterior em uma instituição privada, de julho de 2014 a junho de 2016. Foram excluídos os pacientes que se submeteram à cirurgia de revisão ou que realizaram outra reconstrução ligamentar concomitante. A análise estatística descritiva foi usada para apresentar os resultados. Foi avaliada ainda a associação entre lesão meniscal e as seguintes variáveis: sexo, idade, índice de massa corporal e tempo de lesão. Resultados: Durante o período do estudo, 500 pacientes foram incluídos. Destes, $72,6 \%$ são do sexo masculino. A média da idade foi de 35,1 anos. A média da idade entre as mulheres foi maior do que entre os homens $(37,3 \pm 12,1$ versus $34,3 \pm 10,8)$. O tipo mais comum de fixação femoral e tibial foi a fixação suspensória $(60,8 \%)$ e o parafuso de interferência (96\%), respectivamente. O tendão flexor foi o enxerto mais usado $(70,2 \%)$, seguido pelo tendão patelar $(28,8 \%)$. O aloenxerto foi usado em apenas um caso (0,2\%). A lesão de menisco foi identificada em $44,8 \%$ dos casos, com o menisco medial sendo o mais lesionado (59,4\% das lesões) e a lesão longitudinal sendo a mais frequente $(50,6 \%)$. A meniscectomia parcial foi realizada em $69,5 \%$ dos casos e a sutura de menisco em 14,1\%. O tempo médio de internação hospitalar foi 1,4 dias. Em $25,4 \%$ dos pacientes foi usado um dreno intra-articular após a cirurgia. Não houve nenhum caso de readmissão hospitalar durante o período do estudo. A proporção de homens no grupo de pacientes com lesão de menisco foi significativamente mais alta que no grupo de pacientes sem lesão associada ( $p=0,007)$. Não houve associação entre lesão de menisco e as seguintes variáveis: idade, índice de massa corporal e tempo de lesão. Conclusões: A maioria das reconstruções foram realizadas em homens em todos os grupos de idade, com uma predominância de casos em pacientes acima de 30 anos e com um tempo mediano de lesão de 44 dias. Com relação à técnica cirúrgica, foi encontrada uma baixa taxa de sutura de menisco e alta preferência pelo uso do enxerto de tendões flexores e fixação suspensória no lado femoral. 


\section{INTRODUÇÃO}

A lesão do ligamento cruzado anterior (LCA) é uma lesão comum, especialmente em atletas, além de ser também a lesão ligamentar do joelho que mais frequentemente necessita de reconstrução cirúrgica. ${ }^{(1)}$ Esse tipo de lesão pode trazer sérias consequências para o atleta lesionado e à população em geral devido a um maior risco de osteoartrose precoce, independente do tratamento. ${ }^{(2,3)}$ Ao longo dos anos, a reconstrução do ligamento cruzado anterior (RLCA) tem se tornado um procedimento cirúrgico confiável para restaurar a estabilidade do joelho e prevenir complicações, uma vez que a lesão do LCA predispõe a degeneração condral e meniscal. ${ }^{(4,5)} \mathrm{O}$ número total de RLCA varia de 32 a 78 procedimentos por 100.000 indivíduos/ano e alguns estudos evidenciam que esse número vem aumentando. ${ }^{(1,6-13)}$

A fim de trazer melhorias ao sistema de saúde, muitas instituições e até mesmo países têm desenvolvido registros que funcionam como banco de dados de casos de LCA. Esse tipo de registro é uma ferramenta importante para coletar dados dos pacientes e dos procedimentos realizados, promovendo vários benefícios como a identificação precoce de resultados cirúrgicos ruins causados por um implante ou técnica cirúrgica em particular, bem como a determinação de fatores prognósticos de bons e maus resultados. ${ }^{(7)}$ A RLCA contribui para o aumento dos custos do sistema de saúde e esses custos são ainda maiores quando são incluídos custos indiretos como a reabilitação pós-operatória e os dias de afastamento do trabalho. ${ }^{(6)}$ Sendo assim, os registros poderiam também desempenhar a função de reduzir os custos envolvidos no tratamento da lesão do LCA e ainda fornecer feedback aos hospitais e cirurgiões. ${ }^{(6,7)}$

Com o estabelecimento desses registros, muitos estudos têm focado em analisar tendências cirúrgicas e demográficas da RLCA. A maioria desses estudos tem mostrado que as cirurgias são mais realizadas em adultos jovens, e que a cirurgia é mais realizada em homens do que em mulheres, embora essa diferença pareça estar diminuindo. ${ }^{(6,7,14,15)}$ Esses estudos também mostram que as lesões associadas à lesão do LCA são comuns, tais como as lesões condrais e meniscais. ${ }^{(7,8,13-16)}$ Entretanto, muitos desses estudos foram realizados com dados de sistemas de saúde predominantemente públicos e os hospitais privados podem apresentar uma característica demográfica peculiar em relação à RLCA. ${ }^{(7,13,16,17)}$ Além do mais, poucas informações epidemiológicas estão disponíveis em relação à RLCA em países que não 
têm um registro nacional para coleta de dados e, portanto, algumas variáveis não têm sido bem descritas. A maioria das informações são, portanto, geradas a partir de estudos baseados em questionários respondidos por cirurgiões, os quais são sujeitos a viés. Em virtude disso, os registros de centros acadêmicos podem ser uma boa fonte de dados demográficos e de informações sobre técnicas cirúrgicas desse tipo de procedimento.

\subsection{Objetivos}

1. Descrever as características epidemiológicas dos pacientes submetidos à reconstrução do ligamento cruzado anterior;

2. Descrever as características da prática médica, com foco especial na técnica cirúrgica e cuidados no pós-operatório imediato. 


\section{REVISÃO DA LITERATURA}

A RLCA é um dos procedimentos mais estudados na literatura ortopédica e tem evoluído rapidamente ao longo das últimas décadas, principalmente no que se refere à técnica cirúrgica. ${ }^{(16)}$ Porém, há ainda questões sobre 0 assunto a serem estudadas mas que não podem ser facilmente respondidas com ensaios clínicos randomizados ou meta-análises. ${ }^{(15)}$ Com o alto número de tipos de enxerto e dispositivos de fixação disponíveis, não há ainda um consenso sobre qual deles oferece o melhor resultado clínico. Além do mais, torna-se difícil avaliar todas essas variáveis por meio de ensaios clínicos randomizados. ${ }^{(13)}$ Embora os ensaios clínicos randomizados sejam considerados padrão-ouro como método científico, eles são estudos desenhados para se comparar dois ou poucos métodos de tratamento, tornando-se necessário o uso de outros tipos de estudo como, por exemplo, os registros em larga escala, para se avaliar resultados de múltiplos métodos de tratamento. ${ }^{(13,18)}$ Um ensaio clínico randomizado com o objetivo de demonstrar uma diferença de $5 \%$ nas taxas de revisão após RLCA, por exemplo, necessitaria de quase 500 pacientes em cada grupo; um número muito maior do que é usualmente incluído em ensaios clínicos envolvendo reconstruções ligamentares de joelho. ${ }^{(18)}$ Grandes coortes são, portanto, valiosas para a identificação precoce de procedimentos e dispositivos que resultam em falha precoce, e portanto em resultado funcional inferior. $^{(19)}$

Um registro clínico pode ser definido como um sistema organizado de coleta de informações a respeito do paciente e do procedimento a que este foi submetido, para avaliar resultados específicos para uma população com uma doença ou condição em comum, e que serve a variados propósitos (clínico, científico e de política pública). Para fins de pesquisa científica, registros clínicos são bancos de dados que armazenam informações de um grande número de pacientes e fazem com que os resultados das análises dos dados possam ser generalizados e mais aplicáveis para a população em geral, além de permitir múltiplas comparações de subgrupos, o que não seria facilmente aplicável em um ensaio clínico randomizado. ${ }^{(13,15)}$ Além disso, a diversidade dos pacientes capturados por um registro clínico permite criar uma amostra representativa da população em questão. ${ }^{(15)}$ Há ainda outras vantagens no uso de registros clínicos, como (1) a capacidade de avaliar eventos que não são tão 
comuns (revisão, tromboembolia, infecção do sítio cirúrgico), uma vez que a amostra de pacientes é grande; (2) poder avaliar múltiplos fatores de risco associados com esses eventos; (3) custo reduzido para elaboração de estudos observacionais a partir da análise de dados; (4) coleta de dados realizada de maneira prospectiva e independentemente de objetivos futuros de pesquisa, reduzindo assim o viés de seleção e de informação. ${ }^{(13,15)}$ Contudo, é importante notar que em um estudo de coorte não randomizado envolvendo dados desse tipo de registro clínico, os fatores de confusão devem ser ajustados, ou pela seleção de subgrupos homogêneos ou pelo modelo de regressão múltipla. ${ }^{(7)}$

As partes interessadas em um registro clínico de pacientes são muitas, e elas se beneficiam de diferentes formas. Para o médico, o registro serve para informar dados sobre a apresentação da doença e a prática médica atual no tratamento de determinada condição. Para uma organização médica, os dados do registro podem fornecer informações para avaliar se a comunidade médica tem tratado determinada condição de acordo com as evidências clínicas atuais. Para um paciente, o registro pode aumentar o entendimento da história natural de uma doença. Para a fonte pagadora, o registro pode fornecer informações detalhadas sobre como os procedimentos, dispositivos e produtos farmacêuticos são utilizados em diferentes grupos populacionais, sendo útil para determinar as políticas de cobertura. Para 0 fabricante de uma droga ou dispositivo médico, o registro pode servir para demonstrar a performance do produto em condições reais, desenvolver hipóteses, elaborar ensaios clínicos, identificar e recrutar pacientes que teriam utilidade no desenvolvimento do produto. ${ }^{(20)}$

Os primeiros registros clínicos nacionais foram implantados na Escandinávia, a fim de registrar dados demográficos e cirúrgicos de pacientes submetidos a artroplastias. ${ }^{(21)}$ Esses registros alcançaram sucesso ao gerar dados de performance de múltiplos implantes e técnicas cirúrgicas, identificando precocemente aqueles que contribuem para maus resultados clínicos, e assim evitando um prejuízo para inúmeros pacientes. ${ }^{(13)}$ Baseado na experiência prévia com os registros de artroplastias, surgiram os registros nacionais de LCA também na Escandinávia, com o objetivo de reunir os dados sobre os pacientes e procedimentos realizados, a fim de promover melhorias no tratamento das lesões desse ligamento. ${ }^{(7,13,19)}$ Especificamente, os principais objetivos desses registros são fornecer feedback para os cirurgiões e hospitais, identificar fatores relacionados a bons e maus resultados e identificar 
procedimentos e dispositivos que resultam em falha prematura. ${ }^{(22)}$ Atualmente, os mais importantes registros de LCA do mundo estão disponíveis nos países da Escandinávia e nos Estados Unidos. ${ }^{(18,23,24)}$ Os registros da Escandinávia foram estabelecidos em junho de 2004 na Noruega, em janeiro de 2005 na Suécia e em julho de 2005 na Dinamarca. ${ }^{(18)}$ Todos funcionam de maneira parecida no que se refere à alimentação do banco de dados. Na Noruega, o primeiro país do mundo onde foi estabelecido um registro nacional de LCA, o banco de dados é alimentado prospectivamente com os dados das RLCA realizadas naquele país. Os pacientes que se submetem à cirurgia são convidados a assinarem um termo de consentimento antes do procedimento comunicando-os o tipo de informação que será coletada e também que os mesmos poderão ser convidados futuramente a participar de novos estudos. Além de dados epidemiológicos, o registro contém também dados relativos à técnica cirúrgica que são preenchidos pelo cirurgião imediatamente após o procedimento cirúrgico. Anualmente, os dados descritivos são publicados e enviados para os cirurgiões da associação ortopédica da Noruega, para os hospitais que realizam as reconstruções ligamentares e para as autoridades de saúde responsáveis. Além disso, os dados são individualizados para cada instituição participante e enviados para as mesmas, de maneira que a instituição pode comparar seus dados com os dados nacionais. ${ }^{(7)}$

Além de registros nacionais, há também registros regionais. registro de LCA do Kaiser Permanente é um exemplo de um registro regional, cobrindo uma área de 9,2 milhões de pessoas em 8 diferentes regiões geográficas dos Estados Unidos. A participação é voluntária, porém há um alto índice de aceite por parte do paciente. Em 2010, 93\% das RLCA foram catalogadas pelo registro. ${ }^{(15)}$ Inicialmente, os estudos oriundos da análise de dados desses registros focaram na descrição demográfica da população submetida à reconstrução ligamentar. Apesar disso, a incidência de lesões na população em geral ainda não está bem definida mas, nos Estados Unidos, esta é estimada em 68,6 por 100.000 pessoas/ano. ${ }^{(12)}$ Esse número pode aumentar entre os atletas, visto que esse tipo de lesão comumente ocorre durante a prática de atividade física, que se torna um fator predisponente para lesão. ${ }^{(6,7,16,25)} \mathrm{A}$ incidência de lesão, portanto, depende da população estudada. $\mathrm{Na}$ população geral, a incidência de lesão é mais alta em homens do que em mulheres. ${ }^{(9,12)}$ No entanto, sabe-se que ao estudar apenas a população de atletas que participam de atividades esportivas em nível competitivo, as mulheres apresentam um risco de lesão maior do que os homens. ${ }^{(12)}$ Uma série de fatores têm sido proposta 
para explicar o risco maior entre as mulheres, entre eles a variação hormonal durante o ciclo menstrual e a menor distância intercondilar. ${ }^{(26)}$ Apesar da mais alta incidência em homens na população geral, tem sido relatado um número crescente de RLCA em mulheres ao longo dos anos..$^{(11,12,14,27)}$

A lesão do LCA tem sido descrita como a lesão ligamentar do joelho que mais frequentemente requer tratamento cirúrgico. ${ }^{(1)}$ Nos Estados Unidos tem sido estimado um número de 105.000 RLCA realizadas por ano. ${ }^{(8)}$ Esse número, à semelhança do que ocorre com a incidência da lesão, varia conforme a população e a região estudada. O sistema de saúde e o algoritmo de tratamento adotado pode variar entre os locais estudados e influenciar no número de cirurgias. ${ }^{(23)} \mathrm{Na}$ Suécia, estima-se de acordo com o registro nacional de LCA uma incidência de RLCA de 32 por 100.000 habitantes. ${ }^{(18)}$ Já nos Estados Unidos a incidência anual de reconstruções é de 45 por 100.000 pessoas por ano. ${ }^{(14)}$ A lesão do LCA e sua reconstrução cirúrgica é mais comum nos pacientes jovens, com um pico de lesão ocorrendo em indivíduos entre 19 e 25 anos. ${ }^{(12)}$ Na Noruega, a incidência de RLCA no grupo de 16 a 39 anos é de 85 por 100.000 habitantes. $^{(7)}$ Nessa população, a RLCA tem se tornado um procedimento confiável visando restaurar a estabilidade do joelho em virtude dos bons resultados funcionais após a cirurgia, além de atuar na prevenção de lesões futuras de cartilagem e menisco. ${ }^{(4,5,16,19)}$ Historicamente, a lesão em pacientes mais velhos tem sido tratada sem a realização de cirurgia. A principal razão para essa escolha é a preocupação com complicações pós-operatórias como a trombose venosa profunda, rigidez pósoperatória e artrofibrose, embora não tenha sido descrito um risco aumentado de tais complicações nessa população em comparação com a população mais jovem. ${ }^{(28)}$ À semelhança do que ocorre com os pacientes mais jovens, vários estudos têm evidenciado os bons resultados da RLCA também em pacientes mais velhos. A incidência de lesão e o número de RLCA vem aumentando na população acima de 40 anos, uma vez que os indivíduos dessa faixa etária vem adotando cada vez mais estilos de vida mais saudáveis e praticando atividade física, até mesmo de alta performance, o que leva a um maior risco de lesão e a uma maior escolha pelo tratamento cirúrgico, a fim de manterem-se ativos na prática esportiva. ${ }^{(12,29)} \mathrm{Um}$ melhor entendimento dos aspectos epidemiológicos da lesão do LCA é vital para o desenvolvimento de estratégias de prevenção e tratamento desse tipo de lesão. $O$ objetivo final de todo estudo epidemiológico é ajudar na prevenção da doença ou lesão por meio do conhecimento do paciente sob risco. ${ }^{(11)}$ 
Certos aspectos técnicos da reconstrução cirúrgica do LCA, incluindo posição do túnel femoral, tipo de enxerto e fixação do enxerto permanecem ainda controversos e, portanto, são de interesse da comunidade ortopédica visto que eles podem alterar os resultados clínicos. ${ }^{(30)} \mathrm{A}$ técnica cirúrgica tem evoluído com o passar do tempo em virtude do desenvolvimento dos dispositivos utilizados na cirurgia e da pesquisa a respeito da anatomia e biomecânica do LCA. Similarmente, modificações significantes têm ocorrido nos métodos de reabilitação do LCA. ${ }^{(31)}$ Entretanto, dados a respeito da preferência atual dos cirurgiões no que diz respeito aos detalhes que envolvem a cirurgia, ainda são limitados. Os estudos sobre a prática médica na RLCA permanecem ainda muito restritos às investigações baseadas em questionários enviados aos cirurgiões, ficando sujeitos aos vieses de seleção e lembrança. Mais uma vez, registros ortopédicos tornam-se, nesse sentido, uma boa fonte de informação sobre mudanças na técnica cirúrgica e dispositivos utilizados ao longo dos anos, além de servir como base para pesquisas futuras. ${ }^{(30)}$ 
3 MÉTODOS

\subsection{Registro institucional do ligamento cruzado anterior}

O registro institucional de LCA é um banco de dados alimentado por um protocolo preenchido pelo cirurgião após o procedimento de RLCA na instituição. O protocolo consiste em três partes. A primeira parte compreende informações gerais sobre os dados demográficos do paciente, que inclui sexo, idade, índice de massa corporal (IMC), tempo de lesão até a realização da cirurgia e lesões associadas. As lesões associadas pelo protocolo incluem as lesões de menisco, cartilagem e outras lesões ligamentares que foram tratadas cirurgicamente no momento do procedimento de RLCA. A segunda parte inclui informações relativas ao período intraoperatório. Dados relacionados à técnica cirúrgica, como tipo de enxerto utilizado, dispositivo de fixação do enxerto, tipo de anestesia, tempo total de cirurgia e uso de antibióticos são anotados. Todos os outros procedimentos realizados juntamente com a RLCA, incluindo ressecção ou reparo do menisco e tratamento da lesão condral são anotadas na primeira parte do protocolo. A terceira parte contém informações sobre os cuidados no pós-operatório. Esta seção corresponde ao uso de antibiótico pós-operatório, profilaxia de trombose venosa profunda, uso de dreno pós-operatório e tempo de internação.

\subsection{Análise retrospectiva dos dados}

Uma análise retrospectiva do registro de LCA da instituição entre julho de 2014 e junho de 2016 foi conduzido para coleta de dados. Foram incluídos no estudo todos os pacientes que se submeteram à reconstrução primária do LCA nesse período. Na ausência de dados relevantes no registro, foi então realizada uma análise no prontuário do paciente para a coleta da informação necessária. Um único investigador conduziu a pesquisa dessas informações. Foram excluídos da pesquisa os pacientes com dados insuficientes, cirurgias de revisão de LCA e casos em que foram realizados procedimentos ligamentares concomitantes à RLCA. 


\subsection{Análise estatística}

A análise descritiva (média e desvio-padrão ou mediana e intervalo interquartil, valores mínimo e máximo, frequência absoluta e relativa) foi usada na avaliação dos dados a fim de apresentá-los de forma concisa. A associação entre lesão meniscal e sexo dos pacientes foi analisada pelo teste do qui-quadrado. Dependendo da distribuição observada, o teste $t$ de Student ou o teste não paramétrico de MannWhitney foi usado para avaliar a associação entre lesão de menisco e as seguintes variáveis: idade, sexo, IMC e tempo de lesão. A análise estatística foi realizada usando o software SPSS para Windows. O nível de significância adotado foi 5\%.

\subsection{Aspectos éticos}

A realização do estudo foi aprovada pelo Comitê de Ética em Pesquisa do Hospital Israelita Albert Einstein, sob 0 número CAAE 57919016.0.0000.0071. Os dados foram analisados de forma anônima e os resultados foram apresentados de forma agregada, não permitindo a identificação dos participantes da pesquisa. Não houve custo ou apoio financeiro relacionados aos pesquisadores ou aos sujeitos da pesquisa. 


\section{RESULTADOS}

Um total de 500 pacientes (operados por 93 cirurgiões diferentes) submetidos à RLCA foram incluídos no estudo; $72,6 \%$ foram do sexo masculino e $27,4 \%$ do sexo feminino, com uma média de idade de 35,1 anos ( $\pm 11,2$ anos). Os dados demográficos são apresentados na tabela 1. Foi observado que pacientes da categoria de idade entre 30 e 39 anos apresentaram uma maior prevalência na amostra ( $n=32,2 \%$ ). Uma distinta distribuição com relação ao sexo do pacientes e a idade foi observada; as cirurgias foram mais realizadas em homens na categoria entre 30 e 39 anos, enquanto as mulheres foram mais prevalentes na categoria entre 40 e 49 anos (Figura 1).

Tabela 1. Dados demográficos da amostra

\begin{tabular}{|c|c|c|c|}
\hline Dados demográficos & $\begin{array}{c}\text { Feminino } \\
\mathrm{n}=137\end{array}$ & $\begin{array}{c}\text { Masculino } \\
\mathrm{n}=363\end{array}$ & $\begin{array}{l}\text { Total } \\
\mathrm{n}=500\end{array}$ \\
\hline \multicolumn{4}{|l|}{ Idade (anos) } \\
\hline Média (DP) & $37,3(12,1)$ & $34,3(10,8)$ & $35,1(11,2)$ \\
\hline Mínimo - máximo & $12-64$ & $11-67$ & $11-67$ \\
\hline \multicolumn{4}{|l|}{ Idade (anos) $n(\%)$} \\
\hline 10 a 19 & $17(12,4)$ & $32(8,8)$ & $49(9,8)$ \\
\hline 20 a 29 & $18(13,1)$ & $93(25,6)$ & $111(22,2)$ \\
\hline 30 a 39 & $36(26,3)$ & $125(34,4)$ & $161(32,2)$ \\
\hline 40 a 49 & $42(30,7)$ & $80(22)$ & $122(24,4)$ \\
\hline 50 a 59 & $23(16,8)$ & $30(8,3)$ & $53(10,6)$ \\
\hline 60 a 69 & $1(0,7)$ & $3(0,8)$ & $4(0,8)$ \\
\hline \multicolumn{4}{|l|}{ Índice de massa corporal $\left(\mathrm{kg} / \mathrm{m}^{2}\right)$} \\
\hline Média (DP) & $23,6(3,6)$ & $26,4(3,6)$ & $25,7(3,8)$ \\
\hline Mínimo - máximo & $18,5-44,0$ & $15,5-40,9$ & $15,6-44,1$ \\
\hline \multicolumn{4}{|l|}{$\begin{array}{l}\text { Tempo entre a Lesão e a cirurgia } \\
\text { (dias) }^{\S}\end{array}$} \\
\hline Mediana (IIQ) & $41(20-76)$ & $46(23-96)$ & $44(21-91)$ \\
\hline Mínimo - máximo & $3-991$ & $0-2572$ & $0-2572$ \\
\hline Número de pacientes & 86 & 204 & 290 \\
\hline \multicolumn{4}{|l|}{ Lesão meniscal associada $\mathrm{n}(\%)$} \\
\hline Não & $89(64,9)$ & $187(51,5)$ & $276(55,2)$ \\
\hline $\operatorname{Sim}$ & $48(35,1)$ & $176(48,5)$ & $224(44,8)$ \\
\hline \multicolumn{4}{|c|}{ Lado da lesão meniscal associada n(\%) } \\
\hline Não & $89(65)$ & $187(51,5)$ & $276(55,2)$ \\
\hline Lateral & $16(11,7)$ & $60(16,5)$ & $76(15,2)$ \\
\hline Medial & $29(21,2)$ & $94(25,9)$ & $123(24,6)$ \\
\hline Ambos os meniscos & $3(2,1)$ & $22(6,1)$ & $25(5,0)$ \\
\hline
\end{tabular}

Variáveis categóricas apresentadas por frequências absolutas e relativas (\%); DP: Desviopadrão; §: 210 pacientes excluídos: dados insuficientes 


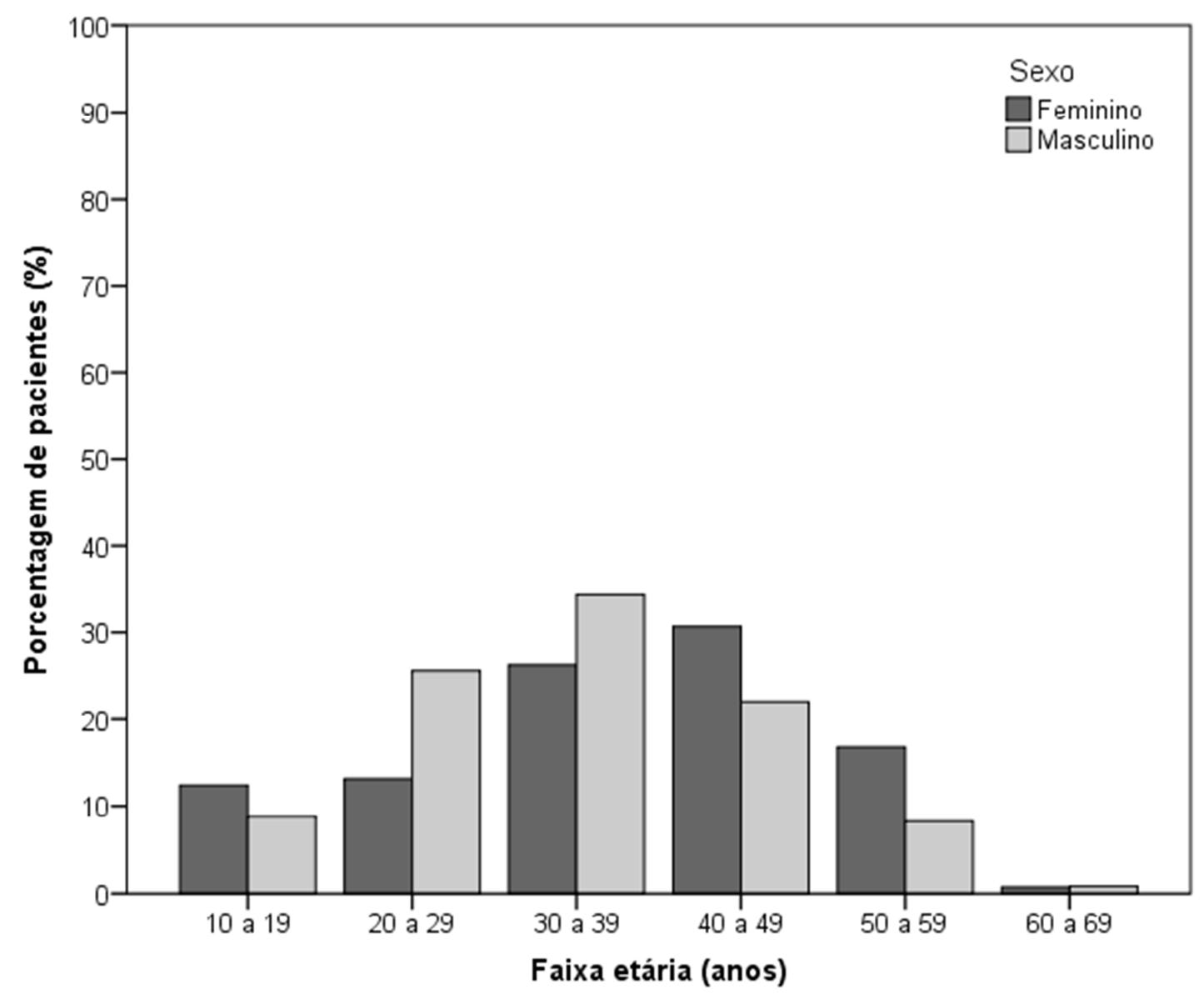

Figura 1. Distribuição por idade de acordo com o sexo dos pacientes

Um total de $351(70,2 \%)$ e $144(28,2 \%)$ RLCA foram realizadas usando tendões flexores (TF) e ligamento patelar (LP) como enxerto, respectivamente. O aloenxerto foi usado em apenas um $(0,2 \%)$ procedimento. Do total de 93 cirurgiões responsáveis pelos casos, apenas 17 optaram por realizar o LP em pelo menos algum procedimento. Os dois dispositivos de fixação mais comumente utilizados foram a fixação suspensória $(60,8 \%)$, seguido pelo parafuso de interferência (34\%). O parafuso de interferência foi utilizado como fixação tibial do enxerto na imensa maioria dos casos (96\%) (Tabela 2). 
Tabela 2. Informações intraoperatórias

\begin{tabular}{|c|c|}
\hline Dados intraoperatórios & $\mathrm{n}(\%)$ \\
\hline \multicolumn{2}{|l|}{ Enxerto } \\
\hline Tendões flexores & $351(70,2)$ \\
\hline Ligamento patelar & $144(28,8)$ \\
\hline Tendão do quadríceps & $4(0,8)$ \\
\hline Aloenxerto & $1(0,2)$ \\
\hline \multicolumn{2}{|l|}{ Tipo de fixação tibial } \\
\hline Parafuso de interferência (metal ou absorvível) & $480(96)$ \\
\hline Fixação suspensória & $10(2,0)$ \\
\hline Parafuso de interferência + fixação secundária & $9(1,8)$ \\
\hline Agrafe & $1(0,2)$ \\
\hline \multicolumn{2}{|l|}{ Tipo de fixação femoral } \\
\hline Fixação suspensória & $304(60,8)$ \\
\hline Parafuso de interferência (metal ou absorvível) & $170(34,0)$ \\
\hline Pino transverso & $24(4,8)$ \\
\hline Fixação suspensória + fixação secundária & $2(0,4)$ \\
\hline \multicolumn{2}{|l|}{ Tipo de anestesia } \\
\hline Raquianestesia & $433(85,2)$ \\
\hline Anestesia geral & $34(6,8)$ \\
\hline Outros & $14(4,2)$ \\
\hline Desconhecida & $19(3,8)$ \\
\hline \multicolumn{2}{|l|}{ Escore ASA } \\
\hline I & $367(73,4)$ \\
\hline II & $106(21,2)$ \\
\hline Desconhecido & $27(5,4)$ \\
\hline \multicolumn{2}{|l|}{ Antibiótico profilático } \\
\hline Sim & $500(100,0)$ \\
\hline \multicolumn{2}{|l|}{ Tipo de antibiótico profilático } \\
\hline Cefazolina & $385(77,0)$ \\
\hline Cefuroxima & $86(17,2)$ \\
\hline Outros & $28(5,6)$ \\
\hline Desconhecido & $1(0,2)$ \\
\hline \multicolumn{2}{|l|}{ Tempo entre antibiótico e cirurgia $(\min )^{\S}$} \\
\hline Mediana (IIQ) & $25(15-30)$ \\
\hline $\operatorname{Min}-\max$ & $-270-730$ \\
\hline Número de pacientes & 426 \\
\hline \multicolumn{2}{|c|}{ Administração do antibiótico até 60 minutos antes da incisão } \\
\hline Sim & $447(89,4)$ \\
\hline Não & $32(6,4)$ \\
\hline Desconhecido & $21(4,2)$ \\
\hline
\end{tabular}

Variáveis categóricas apresentadas por frequências absolutas e relativas (\%); ASA: American Society Anesthesiology; §: 74 pacientes excluídos: ausência dos dados

A lesão meniscal foi identificada em 224 casos (44,8\%); 84,3\% dos casos foram tratados com algum tipo de procedimento e os demais foram deixados in situ. Meniscectomia parcial foi o procedimento mais comum concomitante realizado $(69,5 \%)$, enquanto a sutura meniscal foi realizada em $14,1 \%$ da lesões meniscais; de 71 cirurgiões que se depararam com algum tipo de lesão de menisco durante 0 procedimento, 21 optaram por realizar a sutura da lesão. Houve mais lesões isoladas de menisco medial $(24,6 \%)$ do que lesões isoladas do menisco lateral $(15,2 \%)$; quanto ao tipo de lesão meniscal, as lesões longitudinais foram as mais frequentes $(50,6 \%)$; 
$78,3 \%$ das lesões meniscais foram localizadas no corno posterior do menisco, ou isoladas ou associadas com lesões no corpo e corno anterior do menisco (Tabela 3).

Tabela 3. Informações sobre lesão meniscal e tipo de tratamento

\begin{tabular}{lc}
\hline & Dados sobre lesão meniscal \\
\hline Morfologia & $\mathbf{n}(\%)$ \\
Longitudinal & $126(50,6)$ \\
Complexa & $50(20,1)$ \\
Radial & $23(9,2)$ \\
Flap & $16(6,4)$ \\
Horizontal & $10(4,0)$ \\
Desconhecida & $24(9,6)$ \\
Menisco com lesão & \\
Lateral & $101(40,6)$ \\
Medial & $148(59,4)$ \\
Localização & \\
Corno anterior & $19(13,6)$ \\
Corno posterior & $180(78,3)$ \\
Corpo & $90(42,1)$ \\
Procedimento realizado & $10(4,0)$ \\
Desconhecido & $29(11,6)$ \\
Observação & $210(84,3)$ \\
Sim & $10(4,0)$ \\
Tratamento realizado & $173(69,5)$ \\
Desconhecido & $2(0,8)$ \\
Meniscectomia parcial & $35(14,1)$ \\
Meniscectomia total & $29(11,6)$ \\
Reparo meniscal &
\end{tabular}

Variáveis categóricas apresentadas por frequências absolutas e relativas (\%)

Em relação aos cuidados no pós-operatório imediato, todos os pacientes receberam antibiótico profilático; mais da metade dos casos $(56,6 \%)$ usaram algum tipo de profilaxia tromboembólica; em $25,4 \%$ dos pacientes foi utilizado algum dreno após a cirurgia. As cirurgias em que foram utilizados drenos pós-operatório se distribuíram entre 16 cirurgiões, sendo um único cirurgião responsável por 102 procedimentos $(20,4 \%)$. Uma máquina de movimento passivo contínuo foi usada por quase um quarto dos pacientes $(20,2 \%)$ e a crioterapia foi usada na vasta maioria dos pacientes $(70,2 \%)$. O tempo médio de internação foi 1,4 dias (Tabela 4$)$. Nenhum paciente do estudo teve algum tipo de complicação relacionada ao procedimento que necessitou de nova internação hospitalar no período da análise dos dados. 
Tabela 4. Informações do período pós-operatório imediato

\begin{tabular}{|c|c|}
\hline Dados do período pós-operatório & $\mathrm{n}(\%)$ \\
\hline \multicolumn{2}{|l|}{ Suspensão do antibiótico profilático em até 24 horas } \\
\hline Não & $122(24,4)$ \\
\hline Sim & $377(75,4)$ \\
\hline Desconhecido & $1(0,2)$ \\
\hline \multicolumn{2}{|l|}{ Profilaxia tromboembólica } \\
\hline Não & $217(43,4)$ \\
\hline $\operatorname{Sim}$ & $283(56,6)$ \\
\hline \multicolumn{2}{|l|}{ Tipo de profilaxia tromboembólica } \\
\hline Mecânica & $232(46,4)$ \\
\hline Farmacológica & $12(2,4)$ \\
\hline Mecânica + farmacológica & $39(7,8)$ \\
\hline \multicolumn{2}{|l|}{ Tromboprofilaxia farmacológica } \\
\hline Enoxaparina & $47(9,4)$ \\
\hline Rivaroxaban & $4(0,8)$ \\
\hline \multicolumn{2}{|l|}{ Radiografia pós-operatória (realizada na sala de cirurgia) } \\
\hline Não & $355(71,0)$ \\
\hline Sim & $140(28,0)$ \\
\hline Desconhecido & $5(1,0)$ \\
\hline \multicolumn{2}{|l|}{ Cuidado pós-operatório } \\
\hline Crioterapia & $351(70,2)$ \\
\hline Movimento passivo contínuo & $101(20,2)$ \\
\hline \multicolumn{2}{|l|}{ Dreno intra-articular } \\
\hline Não & $373(74,6)$ \\
\hline Sim & $127(25,4)$ \\
\hline \multicolumn{2}{|l|}{ Tempo de cirurgia (min) } \\
\hline Mediana (IIQ) & $90(75-110)$ \\
\hline $\operatorname{Min}-\max$ & $40-280$ \\
\hline Número de pacientes & 475 \\
\hline \multicolumn{2}{|l|}{ Tempo de internação (dias) } \\
\hline Média (DP) & $1,4(2,1)$ \\
\hline Mediana (IIQ) & $1(1-2)$ \\
\hline Min $-\max$ & $0-35$ \\
\hline
\end{tabular}

Variáveis categóricas apresentadas por frequências absolutas e relativas (\%); DP: desvio-padrão

Houve evidência de associação entre lesão meniscal e sexo dos pacientes $(\mathrm{p}=0,007)$, onde a proporção de homens no grupo de pacientes com lesão meniscal associada $(78,6 \%)$ foi maior do que no grupo de pacientes sem lesão meniscal $(67,8 \%)$. Não encontramos diferença entre os grupos com e sem lesão meniscal em relação à média de idade $(p=0,056)$, IMC $(p=0,206)$ e tempo de lesão $(p=0,384)$ (Tabela 5). 
Tabela 5. Associação entre lesão meniscal e as seguintes variáveis: sexo, idade, índice de massa corporal e tempo de lesão

\begin{tabular}{|c|c|c|c|}
\hline \multirow{2}{*}{ Variável } & \multirow{2}{*}{ Total $(n=500)$} & \multicolumn{2}{|c|}{ Lesão meniscal } \\
\hline & & Não (n=276) & $\operatorname{Sim}(n=224)$ \\
\hline \multicolumn{4}{|l|}{ Sexo $n(\%)$} \\
\hline Feminino & $137(27,4 \%)$ & 89 (32,2\%) & $48(21,4 \%)$ \\
\hline \multirow{2}{*}{ Masculino } & 363 (72,6\%) & $187(67,8 \%)$ & $176(78,6 \%)$ \\
\hline & & \multicolumn{2}{|c|}{ Teste de chi-quadrado: $p=0,007$} \\
\hline \multicolumn{4}{|l|}{ Idade (anos) } \\
\hline Média (DP) & $35,1(11,2)$ & $34,2(10,5)$ & $36,2(12,0)$ \\
\hline \multirow[t]{2}{*}{ Min - max } & $11-67$ & $11-59$ & $12-67$ \\
\hline & & \multicolumn{2}{|c|}{ Teste $t$ de Student: $p=0,056$} \\
\hline \multicolumn{4}{|l|}{ IMC (kg/m²) } \\
\hline Média (DP) & $25,7(3,8)$ & $25,5(3,8)$ & $25,9(3,9)$ \\
\hline \multirow{2}{*}{ Min - max } & $15,6-44,1$ & $18,5-40,0$ & $15,6-44,1$ \\
\hline & & \multicolumn{2}{|c|}{ Teste $t$ de Student's: $\mathrm{p}=0,206$} \\
\hline \multicolumn{4}{|c|}{ Tempo de lesão (dias) $)^{\S}$} \\
\hline Mediana (Q1; Q3) & $44,0(21,0 ; 91,0)$ & $45,5(23,5 ; 95,5)$ & $42,0(20,0 ; 87,0)$ \\
\hline $\operatorname{Min}-\max$ & $0-2572$ & $5-991$ & $0-2572$ \\
\hline $\mathrm{n}$ & 290 & 156 & 134 \\
\hline & & \multicolumn{2}{|c|}{ Teste $t$ de Student's: $\mathrm{p}=0,384$} \\
\hline
\end{tabular}

Variáveis categóricas apresentadas por frequências absolutas e relativas (\%); DP: desvio-padrão; Q1: primeiro quartil; Q3: terceiro quartil; IMC: índice de massa corporal

$\S: 210$ pacientes excluídos: dados insuficientes 


\section{DISCUSSÃO}

Esse estudo descreve as características da prática médica além de descrever a epidemiologia da RLCA usando dados de um hospital privado situado no Brasil. Nosso estudo focou primariamente nas características epidemiológicas dos pacientes submetidos à cirurgia, achados intraoperatórios e aspectos técnicos da cirurgia. Esses achados podem contribuir para um melhor entendimento da epidemiologia e tendências na RLCA em nosso meio.

Nosso estudo mostrou que houve um maior número de RLCA em homens em comparação às mulheres em todos os grupos de idade. Provavelmente, este achado é relacionado à prática de esporte, considerando que no Brasil, os homens praticam mais esportes associados com lesão do LCA do que as mulheres. $E$, de fato, a maioria das lesões de LCA ocorre durante a prática de esportes. ${ }^{(6,7,16,25)}$ Muitos estudos têm relatado que a incidência de lesão de LCA é maior entre homens do que em mulheres na população em geral. ${ }^{(9,12)}$ Embora muitos autores relatem esta predominância de lesão entre homens, parece que esta diferença vem diminuindo com o tempo. ${ }^{(11,12,14,27)}$ Um estudo usando dados do sistema de saúde americano, mostrou que as mulheres representavam 32\% das RLCA realizadas em 1994 e aumentou para $42 \%$ em 2004. ${ }^{(11)}$ Isto provavelmente reflete uma participação crescente das mulheres em esportes que exijam movimentos rotacionais do joelho, aumentando o risco de lesão de LCA. Além disso, vários estudos têm mostrado que as mulheres apresentam maior risco de lesão do LCA em comparação com os homens, quando participam de esportes competitivos. ${ }^{(32)}$

Em nosso estudo, a média de idade dos pacientes submetidos a RLCA foi superior a de outros estudos. ${ }^{(8,11,12,14-16,25)}$ Interessantemente, a média de idade em mulheres foi ainda maior do que nos homens. Nordenvall et al. ${ }^{\left({ }^{9}\right)}$ relataram que as mulheres são lesionadas em uma idade mais precoce que os homens, com um pico de incidência entre 11 e 20 anos. Nós observamos que uma maior proporção de indivíduos tinha mais de 30 anos e quase metade das mulheres da amostra tinha mais de 40 anos. Isto está de acordo com os resultados de um estudo realizado nos Estados Unidos que mostrou um aumento no número de RLCA em pacientes com mais de 40 anos. ${ }^{(11)}$ Provavelmente, as razões para esta tendência são os bons resultados funcionais após a RLCA em pacientes mais velhos e o menor risco de revisão e 
reconstrução cirúrgica contralateral conforme o aumento da idade, os quais tem sido relatados por estudos recentes. ${ }^{(29,33)}$ Além disso, Seng et al. ${ }^{(34)}$ sugeriram que pacientes mais velhos estão preferindo o tratamento cirúrgico para manterem-se funcionalmente capazes de realizar algum tipo de atividade física. Por outro lado, parece haver uma relação entre idade e readmissão hospitalar após a cirurgia de RLCA. Um estudo que avaliou a taxa de readmissão após a RLCA demonstrou que pacientes com mais de 40 anos foram readmitidos com mais frequência do que pacientes mais jovens, independentemente de outros fatores. ${ }^{(8)}$

As cirurgias realizadas em caráter ambulatorial são benéficas em muitos aspectos, como a redução de custos $^{(35)}$ e a maior satisfação do paciente ${ }^{(36)}$ comparado com o paciente operado em caráter hospitalar. Além disso, um estudo americano relatou que as taxas de readmissão hospitalar dentro de 90 dias após a RLCA foi significativamente maior entre pacientes que operaram em caráter hospitalar. ${ }^{(8)}$ A definição de cirurgia ambulatorial ainda não é muito clara na literatura mas geralmente refere-se ao procedimento realizado no setor ambulatorial do hospital, onde o paciente receberá alta hospitalar no mesmo dia da cirurgia. ${ }^{(37,38)}$ Por conta das inúmeras vantagens, o número de cirurgias para reconstrução do LCA realizadas ambulatorialmente vem aumentando. Nos Estados Unidos, esse tipo de cirurgia subiu de $43 \%$ em 1994 para 95\% em 2006. ${ }^{(11)}$ Vários outros estudos têm relatado um aumento no número de RLCA realizadas ambulatorialmente. ${ }^{(8,14,39,40)}$ Esta tendência também parece estar ocorrendo na Europa. Em um estudo descrevendo os registros de LCA da Escandinávia, foi observado que na Dinamarca $79 \%$ das reconstruções são ambulatoriais. ${ }^{(18)}$ Por outro lado, todos os casos em nosso estudo foram realizados em nível hospitalar. Apesar disso, não houve nenhuma readmissão em nossa amostra. $O$ tempo médio de internação em nosso estudo foi 1,4 dias. Outros estudos que também avaliaram este dado encontraram um resultado similar. Estudo realizado nos Estados Unidos, avaliando apenas as reconstruções realizadas em regime hospitalar de 1998 a 2010, relatou que o tempo médio de internação foi 1,6 dias. ${ }^{(35)}$ Outro estudo similar, porém com dados do sistema de saúde pública do Brasil, encontrou um tempo médio de internação de 1,8 dias em 2014. ${ }^{(41)}$ Nesse mesmo estudo, os autores mostraram uma redução no tempo de internação ao longo dos anos. Por causa dos benefícios e da segurança da RLCA realizada ambulatorialmente, a cirurgia realizada em regime hospitalar deve ser reservada para aqueles pacientes que por alguma razão, seja considerado inseguro receber alta no mesmo dia da cirurgia. ${ }^{(35)}$ 
Foi observada nesse estudo uma alta preferência pelo uso do enxerto de TF. Nosso achado está de acordo com outros estudos que também relatam a atual preferência por este tipo de enxerto. ${ }^{(7,13,16,19,23,25,31,42)}$ Recentemente, tem havido uma tendência em usar mais TF em detrimento do LP. ${ }^{(16,19,40)}$ Dois estudos que usaram o registro de LCA da Suécia relataram que o número de RLCA com o uso de TF tem aumentado desde 2005. ${ }^{(16,19)}$ Em 2010, 96,1\% das RLCA primárias na Suécia foram realizadas usando TF.(19) Além disso, RLCA usando LP vem gradualmente diminuindo. ${ }^{(19)}$ A razão para esta tendência na escolha do enxerto é provavelmente devida a menor morbidade do sítio doador e menor dor pós-operatória observada com o uso de TF comparado com LP. ${ }^{(43)}$ Além disso, há provavelmente uma correlação entre o uso do LP e o desenvolvimento de osteoartrose, o que pode influenciar a escolha do enxerto. ${ }^{(44)} \mathrm{O}$ uso do TF, por sua vez, tem sido associado com um maior risco de revisão precoce. ${ }^{(15,33)}$ Embora esta preferência por um tipo específico de enxerto, uma recente meta-análise não identificou qual dos dois tipos de enxerto é funcionalmente melhor para a RLCA. ${ }^{(43)}$ Outra possível fonte de enxerto é o tendão do quadríceps. Uma grande vantagem deste é que ele pode ser usado sem o plug ósseo com mínima morbidade do sítio doador. ${ }^{(45)}$ Atualmente, este tipo de enxerto é menos usado que os TF e o LP e, portanto, é menos estudado. ${ }^{(15,42)}$ Provavelmente, a maioria dos ortopedistas nem mesmo oferece esta opção de enxerto ao discutir os detalhes cirúrgicos com o paciente. Entretanto, tem havido um interesse crescente no uso do tendão do quadríceps após os bons resultados relatados. ${ }^{(46)}$ Em relação à prevalência do uso de aloenxerto, nossos resultados são similares aos dos registros de LCA da Escandinávia onde o aloenxerto tem sido também menos indicado. ${ }^{(18)}$ Entretanto, 0 Kaiser Permanent Anterior Cruciate Ligament Reconstruction Registry (KPACLRR) e o Multicenter Orthopaedic Outcomes Network (MOON), dois registros localizados nos Estados Unidos, relataram uma alta proporção de uso do aloenxerto $(42,4$ e $13 \%$, respectivamente). ${ }^{(23,30)}$ Este achado vai de encontro ao de outros estudos e poderia ser explicado por aspectos culturais que ainda não foram descritos. De qualquer maneira, a alta taxa de uso do aloenxerto nesses estudos é surpreendente devido aos recentes estudos que relataram um maior risco de revisão precoce e piores resultados funcionais com aloenxerto, principalmente em pacientes jovens. ${ }^{(15,33,47)}$

Vários fatores técnicos da RLCA, dentre eles o método de fixação do enxerto, podem afetar os resultados clínicos. Cirurgiões têm diferentes opções para a fixação do enxerto e todos eles apresentam vantagens e desvantagens. Portanto, a 
fixação do enxerto pode depender do tipo do enxerto, preferência do cirurgião e política do seguro de saúde. Em nosso estudo, a fixação do enxerto usando um dispositivo de fixação suspensória foi o método mais comumente usado no lado femoral $(60,8 \%)$ seguido por parafuso de interferência (34\%). O uso do pino transverso correspondeu a apenas $4 \%$ de todos os casos. O método de fixação no lado femoral tem mudado ao longo dos anos e este fato pode ser explicado provavelmente devido ao conceito de reconstrução anatômica do LCA. ${ }^{(42)}$ Um estudo realizado na Suécia mostrou que pinos transversos foram usados em 60,4\% dos pacientes em 2005 contra apenas 5,3\% em 2012. ${ }^{(16)}$ Estudos mais recentes têm descrito não apenas a diminuição no uso do pino transverso mas também uma mudança para o uso dos dispositivos de fixação suspensória ao invés do pino transverso no lado femoral. ${ }^{(30,40)}$

As lesões de LCA são frequentemente acompanhadas por lesões de menisco. Isto tem sérias consequências para o paciente, uma vez que o procedimento meniscal concomitante à RLCA tem sido associado com cirurgias subsequentes no joelho. ${ }^{(8)}$ Em nosso estudo, a lesão meniscal foi identificada em $44,8 \%$ dos pacientes, uma taxa similar a prevalência relatada por outros estudos. ${ }^{(7,12)}$ Estudos prévios têm relatado a incidência de lesões meniscais concomitantes em taxas que variam de 35 a 92,8\%. ${ }^{(4,15,18,25,27,48)}$ Tem também sido descrito uma alta prevalência de lesão associada de menisco medial comparada com lesão de menisco lateral. ${ }^{(5,23,34,47)}$ Embora alguns estudos relatem a incidência de lesões meniscais durante a RLCA, poucos estudos descrevem o tipo de lesão meniscal e sua localização dentro do menisco. Em um estudo prospectivo com 541 pacientes submetidos a RLCA, foi relatado que o corno posterior de ambos os meniscos foi o local mais comumente lesionado. ${ }^{(5)}$ Outro estudo relatou achados similares, com as lesões periféricas do corno posterior do menisco medial sendo o tipo mais comum de lesão em pacientes com lesão do LCA. ${ }^{(34)} \mathrm{Em}$ concordância com outros estudos, nosso estudo demonstrou que o corno posterior esteve envolvido na maioria dos casos com lesão meniscal e a lesão longitudinal foi o padrão mais comumente encontrado.

A lesão de LCA associada com lesões meniscal ou condral, quando comparada com as lesões isoladas de LCA, são associadas com um maior risco de desenvolvimento de osteoartrose. ${ }^{(49)}$ Em vista disso, os preditores de lesões intraarticulares associados com lesão de LCA têm sido investigados por outros autores. ${ }^{(5,50)}$ Em nosso estudo, houve uma associação entre sexo e lesão meniscal, com homens apresentando uma maior prevalência de lesão meniscal do que as mulheres. Similar ao 
nosso estudo, o sexo masculino tem sido associado com uma maior prevalência de lesão meniscal por estudos prévios. Kluczynski et al.,(5) em um estudo prospectivo, mostraram a associação entre sexo e lesão meniscal mas não entre a lesão meniscal e outras variáveis, como idade, IMC e tempo de lesão. Ageberg et al. ${ }^{(51)}$ também relataram que mulheres têm menos lesão meniscal associada do que homens. Brambilla et al., ${ }^{(50)}$ em um estudo incluindo 988 pacientes, relataram não apenas a associação entre a lesão meniscal com o sexo masculino mas também um maior risco de lesão meniscal um ano após a lesão do LCA. Ao contrário de outros estudos, nós não encontramos correlação entre idade, IMC, tempo de lesão e lesão meniscal.

Com relação ao tempo de lesão, encontramos um valor mediano de 44 (21 a 91) dias. O tempo de lesão pode variar consideravelmente entre os diferentes estudos. Granan et al. ${ }^{(18)}$ relataram um período relativamente longo de tempo entre a lesão e o momento da cirurgia nos registros escandinavos de LCA. Por outro lado, em um estudo envolvendo dados de RLCA de sete centros acadêmicos nos Estados Unidos, o valor mediano de tempo de lesão até o momento da cirurgia foi de 2,4 meses. $^{(23)}$ Dois fatores podem explicar as diferenças encontradas nos estudos. Primeiro, o algoritmo de tratamento para lesão de LCA podem ser diferentes dependendo do país. Magnussen et al. ${ }^{(23)}$ relataram que o tratamento não operatório é mais comum na Noruega do que nos Estados Unidos. Segundo, pode haver maior tempo de espera na realização da cirurgia em alguns países que outros, dependendo do sistema de saúde adotado. Nosso estudo foi realizado em um hospital privado, que pode explicar o período relativamente baixo de tempo de lesão dos pacientes até o momento da cirurgia.

Estudos prévios têm demostrado os benefícios da sutura do menisco em diminuir o risco de osteoartrose, em comparação à meniscectomia. ${ }^{(52,53)}$ A sutura de menisco realizada concomitante com a RLCA tem uma taxa de sucesso maior quando comparada com a sutura isolada. ${ }^{(53)}$ Similar aos nossos achados, vários estudos demonstram que a meniscectomia parcial ainda é o procedimento preferido pelos cirurgiões diante de qualquer tipo de lesão de menisco. ${ }^{(18,23,48,54-56)}$ No entanto, a prevalência de sutura de menisco em nosso estudo se mostrou diferente da taxa relatada por outros autores. Em um estudo que comparou dois registros de LCA, foi identificado que $39 \%$ dos casos registrados no MOON (Registro de LCA situado nos EUA) e $22 \%$ dos casos do Norwegian cruciate ligament register (Registro nacional norueguês) foram tratados com sutura da lesão. ${ }^{(23)}$ Estudos prévios nos Estados 
Unidos relataram um aumento no número de suturas de menisco realizadas no momento da RLCA. ${ }^{(57,58)}$ Isto é provavelmente um reflexo da disseminação da informação a respeito dos efeitos condroprotetores da preservação do menisco. ${ }^{(58)}$ Apesar disso, em um estudo realizado no Brasil, foi demonstrado que menos de $2 \%$ dos cirurgiões, tanto do sistema de saúde público como do privado, realizam rotineiramente a sutura do menisco no mesmo tempo da RLCA. ${ }^{(59)}$ Provavelmente, a baixa taxa de sutura meniscal descrita em nosso estudo pode ser devida à técnica cirúrgica ser mais demandante comparada à meniscectomia, ao maior tempo de recuperação exigido após a sutura e a média de idade mais elevada entre os indivíduos desse estudo. Além disso, a sutura do menisco requer implantes considerados de alto custo. Esses fatores podem desencorajar alguns cirurgiões a realizar tal procedimento. Entretanto, tem havido mudanças tanto na técnica cirúrgica quanto na reabilitação, podendo levar a uma recuperação pós-operatória mais rápida e fazendo da sutura de menisco um procedimento mais confiável para pacientes e cirurgiões. ${ }^{(60)}$ Os 35 casos $(14,1 \%)$ de sutura de menisco foram realizados por 21 cirurgiões diferentes (de 61 cirurgiões que se depararam com uma lesão passível de tratamento). Isso evidencia uma prática média homogênea na instituição, uma vez que o procedimento não se mostrou restrito a poucos profissionais. Ainda pensando em evitar a meniscectomia, sabe-se que alguns tipos de lesão de menisco apresentam maior chance de cicatrização mesmo sem a sua sutura. As lesões que podem seguramente permanecer sem tratamento durante a RLCA são aquelas lesões de espessura parcial do menisco que tenham comprimento menor que 10 milímetros. ${ }^{(61)}$

Com relação aos cuidados no período pós-operatório imediato, poucos estudos trazem informações sobre o uso da crioterapia, uso da máquina de movimentação passiva contínua e uso de dreno intra-articular. Em nosso estudo, nós identificamos que a crioterapia foi usada na vasta maioria dos casos. Este dado é similar aos resultados encontrados por Coskunsu et al. ${ }^{(62)}$ Este resultado pode ser explicado pelos benefícios da crioterapia em reduzir a dor no período pós-cirúrgico imediato já relatado em outros estudos. ${ }^{(63)}$ Com relação ao uso da máquina de movimento passivo contínuo, estudos investigando a preferência dos cirurgiões, têm mostrado taxas no seu uso que variam de 23,4 a $68,6 \% .{ }^{(31,39,40,62)}$ Esses valores surpreendem, uma vez que estudos prévios não mostram benefícios a longo prazo quando comparado com o tratamento padrão, como foi relatado por uma recente revisão. ${ }^{(64)}$ Assim como a máquina de movimento passivo contínuo, o uso rotineiro de 
dreno intra-articular após a RLCA não é recomendada por vários autores. Dhawan et al. ${ }^{(65)}$ mostraram que o uso de dreno após a cirurgia não oferece benefícios em termos de arco de movimento, edema ou dor no período pós-operatório imediato. Outros estudos mostram resultados similares. ${ }^{(66,67)}$ Ainda assim, em nosso estudo, observamos que um quarto dos pacientes usaram dreno. Os 127 procedimentos em que foi utilizado dreno foram realizados por 16 cirurgiões diferentes, evidenciando que a prática do uso de dreno pós-operatório nas RLCA está atualmente restrita a um baixo número de cirurgiões da instituição. De qualquer maneira, estes achados mostram que as opiniões entre os cirurgiões ainda se dividem com relação a alguns aspectos cirúrgicos.

A profilaxia antibiótica em cirurgia ortopédica é efetiva em reduzir as taxas de infecção pós-operatória. ${ }^{(68)}$ A profilaxia deve ser realizada previamente à insuflação do torniquete e grande parte dos estudos recomendam ainda o seu uso dentro do período de uma hora antes da incisão cirúrgica. ${ }^{(68,69)}$ A suspensão do antibiótico é recomendada em até 24 horas, independente do uso de drenos póscirúrgicos. ${ }^{(69)} \mathrm{Em}$ nosso estudo verificou-se que $100 \%$ dos pacientes realizaram antibioticoprofilaxia; 89,4\% realizaram a profilaxia até 60 minutos da incisão cirúrgica e $75,4 \%$ tiveram o antibiótico suspenso em até 24 horas. O uso racional da profilaxia antibiótica deve ser estimulado a fim de aumentar o seu benefício e minimizar os efeitos adversos. Diferente da profilaxia antibiótica, a profilaxia contra trombose venosa profunda (TVP) não é tão padronizada durante a RLCA e, portanto, a decisão de usar ou não algum tipo de profilaxia é influenciada pela experiência clínica de cada cirurgião. ${ }^{(70)}$ Grande parte dos cirurgiões consideram esse tipo de cirurgia como sendo de baixo risco para o desenvolvimento de TVP e, portanto, recomendam apenas a mobilização precoce como método de profilaxia mecânica. ${ }^{(70)}$ Em estudo recente para investigar o uso de tromboprofilaxia nesse tipo de cirurgia, verificou-se que pouco mais da metade dos cirurgiões que foram questionados sobre o tema utilizavam rotineiramente alguma profilaxia química. ${ }^{(70)}$ Nesse mesmo estudo, a medicação preferida foi o ácido acetilsalicílico. Já no nosso estudo, o uso de profilaxia química foi utilizada em apenas $10,2 \%$ dos pacientes e a droga preferida foi a enoxaparina, utilizada em $9,4 \%$ dos pacientes.

A utilidade clínica da radiografia realizada no período pós-operatório imediato em variados procedimentos ortopédicos, muitas vezes ainda na própria sala de cirurgia, vem sendo recentemente questionada por alguns estudos. ${ }^{(71,72)}$ Mais ainda 
em um momento em que muito se discute sobre a custo-efetividade de procedimentos na área da saúde. A RLCA primária é um procedimento ortopédico com uma taxa baixa de complicações e, portanto, a realização de radiografia pós-operatória imediata tornase questionável. Em nosso estudo, demonstramos que em pelo menos $28 \%$ dos procedimentos foi realizada a radiografia pós-operatória ainda na própria sala cirúrgica. Estudos que investigaram pacientes submetidos à RLCA e que realizaram radiografia pós-operatória relataram não haver mudanças no cuidado pós-operatório desses pacientes baseado nos achados radiográficos, não justificando assim o seu uso. ${ }^{(71,72)}$

Nosso estudo apresenta limitação com respeito a natureza dos dados uma vez que se trata de uma análise retrospectiva de banco de dados. Algumas informações clínicas tais como o mecanismo de trauma, presença de lesão condral e protocolo de reabilitação não foi avaliado. Além disso, os dados refletem a amostra de um pequeno número de cirurgiões, e pode ser que o padrão de tratamento observado represente apenas a prática médica de um hospital privado específico. No entanto, esse estudo apresenta relevância clínica ao contribuir provendo informações para a comunidade ortopédica a fim de refinar nosso conhecimento a respeito da RLCA, além de contribuir promovendo melhorias no tratamento das lesões do LCA uma vez que nós temos a oportunidade de conhecer melhor as características dos pacientes e a tendência entre os cirurgiões e, com isso, comparar com as evidências clínicas de melhores resultados já disponíveis na literatura, promovendo dessa maneira uma mudança positiva na prática clínica do tratamento cirúrgico dessas lesões. Por exemplo, com os dados presentes nesse estudo, a instituição pode elaborar um plano visando conscientizar os cirurgiões dos benefícios da sutura do menisco e desaconselhar o uso do dreno intra-articular e da máquina de movimento passivo contínuo, visto que não trazem benefícios a longo prazo para o paciente. No futuro, o registro institucional do LCA pode servir também como base, principalmente após avaliação e seguimento pós-operatório desses pacientes, para determinar prognóstico e preditores de bons ou maus resultados dos pacientes que irão se submeter a cirurgia. 


\section{CONCLUSÕES}

1. As reconstruções do ligamento cruzado anterior foram realizadas principalmente em homens em todos os grupos de idade analisados, com predominância em pacientes acima de 30 anos de idade e com um tempo mediano de lesão de 44 dias.

2. Com relação à técnica cirúrgica, houve uma baixa taxa de sutura de menisco e uma alta preferência pelo uso do enxerto de tendões flexores e pela fixação suspensória como método de fixação femoral. 
7 ANEXOS

Anexo 1. Protocolo preenchido pelo cirurgião após a cirurgia

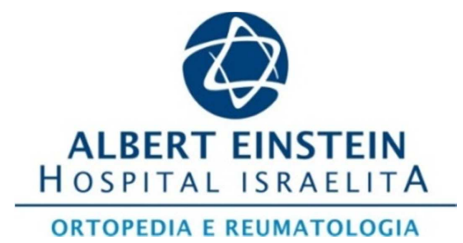

Data do trauma:

Data da cirurgia:

Peso:

Altura:

\section{Diagnóstico inicial}

( $x$ ) Lesão de LCA

Diagnóstico associados:

Assinale e indique no desenho a localização e o tipo de lesão

( ) Lesão do menisco medial

( ) Lesão do Canto Postero Lateral

( ) Lesão do menisco lateral

( ) Lesão condral

( ) Lesão do Ligamento Colateral Medial

( ) Sem lesões associadas
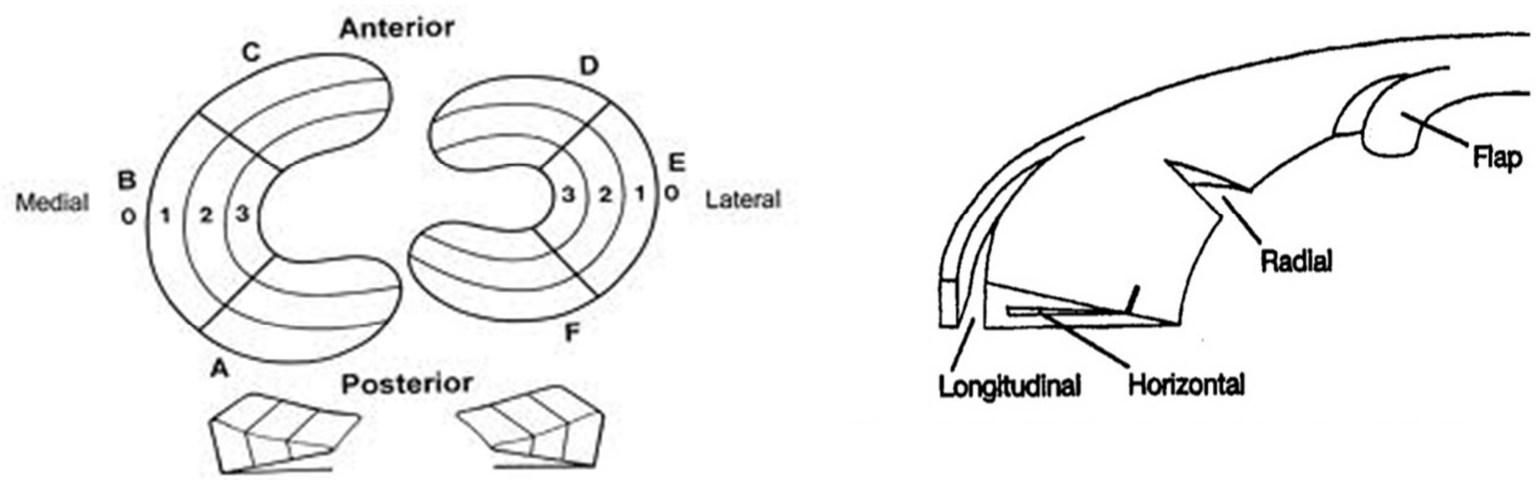
( ) Lesão Longitudinal
( ) Lesão horizontal
( ) Lesão radial

( ) Lesão tipo alça de balde

( ) Lesão degenerativa

( ) Lesão tipo Flap 


\section{Cirurgia do Menisco ( ) Sim （） Não}

( ) Sutura de menisco

( ) Meniscectomia Parcial ( ) Total ( )

( ) Somente observar resultado

\section{Lesão condral}

( ) Côndilo femoral medial

Tamanho:___ $\mathrm{cm}^{2}$

( ) Côndilo femoral lateral

Tamanho: $\quad \mathrm{cm}^{2}$

( ) Troclea central

Tamanho: $\quad \mathrm{cm}^{2}$

( ) Troclea lateral

Tamanho: $\quad \mathrm{cm}^{2}$

( ) Troclea medial

Tamanho: $\quad \mathrm{cm}^{2}$

( ) Patela faceta lateral

Tamanho: $\quad \mathrm{cm}^{2}$

( ) Patela Faceta medial

Tamanho: $\quad \mathrm{cm}^{2}$

( ) Patela vértice

Tamanho:__ $\mathrm{cm}^{2}$

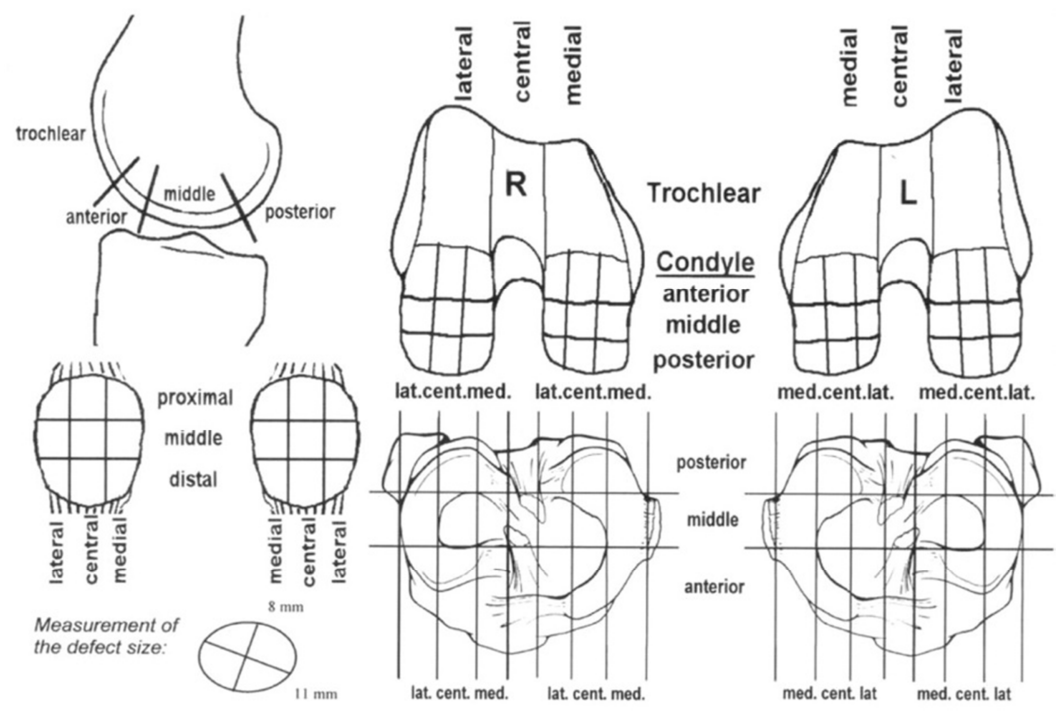

Desenhe o local e o tamanho da lesão

\section{Cirurgia}
( ) Abrasão
( ) Mosaicoplastia
( ) Microfratura
( ) Revisão
( ) Reconstrução extra articular associada
( ) Reconstrução extra articular isolada
( ) Outros: 


\section{Tipo de Enxerto}
( ) Flexor
( ) Tendão patelar
( ) Tendão quadriciptal
( ) Aloenxerto

Fixação

( ) Tíbia

( ) Fêmur

Diâmetro do enxerto:

\section{Comorbidades}

( ) Hipertensão arterial sistêmica

( ) Tabagismo

( ) Diabetes mellitus

( ) Infecção local

( ) Trombose venosa profunda prévia

\section{INTRAOPERATÓRIO}

Cirurgião:

1ㅇ Auxiliar:

Anestesista:

\section{Fabricante:}

( ) J\& J

( ) Imact

( ) Bone Surgical

( ) PCE

( ) Tellus

( ) Outros:

Tipo de Anestesia :

Critério Asa: ( ) I ( ) II ( ) III ( ) IV

Potencial de contaminação:

\section{Prevenção de infecção}

Infecção previa ( ) Sim ( ) Não

Em uso de antibioticoterapeutico antes da cirurgia ( ) Sim ( ) Não

Inicio da cirurgia............. Termino da cirurgia............ 
Tempo total.........

Horário do ATB profilático

Recebeu antibióticoprofilático nos 60 minutos antes da cirurgia:

( ) $\operatorname{Sim}($ ) Não

Qual: ( ) Cefazolina (Kefazol) dose:

( ) Cefalotina (Keflin) dose:

( ) Cefuroxima (Zinacef) dose:

( ) Outros

Suspensão em 24h ( ) Sim ( ) Não

Data da ultima dose........./....

Horário da ultima dose........

\section{PÓS-OPERATÓRIO}

Radiografia ou Radioscopia pós- Op. ( )Sim ( )Não

\section{Profilaxia para TVP:}

( ) Mecânica (compressor)

( ) Meias elásticas

( ) Medicamentosa

( ) Enoxaparina ( ) 20mg/sc/1x/dia ( ) 40mg/sc/1x/dia

( ) $60 \mathrm{mg} / \mathrm{sc} / 1 \mathrm{x} / \mathrm{dia}$

Heparina sódica: dose

Iniciou a tromboprofilaxia medicamentosa até 24 horas após o procedimento cirúrgico?

( ) Sim ( ) Não

\section{Meta em 24 horas:}

( ) Crioterapia

( ) Cpm

( ) Deambular

Alta

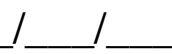




\section{REFERÊNCIAS}

1. Gianotti SM, Marshall SW, Hume PA, Bunt L. Incidence of anterior cruciate ligament injury and other knee ligament injuries: a national population-based study. J Sci Med Sport. 2009;12(6):622-7.

2. Andersson D, Samuelsson K, Karlsson J. Treatment of anterior cruciate ligament injuries with special reference to surgical technique and rehabilitation: an assessment of randomized controlled trials. Arthroscopy. 2009;25(6):653-85. Review.

3. Riccardo C, Fabio C, Pietro R. Knee osteoarthritis after reconstruction of isolated anterior cruciate ligament injuries: a systematic literature review. Joints. 2017;5(1):39-43. Review.

4. Ghodadra N, Mall NA, Karas V, Grumet RC, Kirk S, McNickle AG, et al. Articular and meniscal pathology associated with primary anterior cruciate ligament reconstruction. J Knee Surg. 2013;26(3):185-93.

5. Kluczynski MA, Marzo JM, Bisson LJ. Factors associated with meniscal tears and chondral lesions in patients undergoing anterior cruciate ligament reconstruction: a prospective study. Am J Sports Med. 2013;41(12):2759-65.

6. Janssen KW, Orchard JW, Driscoll TR, van Mechelen W. High incidence and costs for anterior cruciate ligament reconstructions performed in Australia from 2003-2004 to 2007-2008: time for an anterior cruciate ligament register by Scandinavian model? Scand J Med Sci Sports. 2012;22(4):495-501.

7. Granan LP, Bahr R, Steindal K, Furnes O, Engebretsen L. Development of a national cruciate ligament surgery registry: the Norwegian National Knee Ligament Registry. Am J Sports Med. 2008;36(2):308-15.

8. Lyman S, Koulouvaris P, Sherman S, Do H, Mandl LA, Marx RG. Epidemiology of anterior cruciate ligament reconstruction: trends, readmissions, and subsequent knee surgery. J Bone Joint Surg Am. 2009;91(10):2321-8.

9. Nordenvall R, Bahmanyar S, Adami J, Stenros C, Wredmark T, Felländer-Tsai L. A population-based nationwide study of cruciate ligament injury in Sweden, 2001-2009: incidence, treatment, and sex differences. Am J Sports Med. 2012;40(8):1808-13.

10. Csintalan RP, Inacio MC, Funahashi TT. Incidence rate of anterior cruciate ligament reconstructions. Perm J. 2008;12(3):17-21. 
11. Mall NA, Chalmers PN, Moric M, Tanaka MJ, Cole BJ, Bach BR Jr, et al. Incidence and trends of anterior cruciate ligament reconstruction in the United

States. Am J Sports Med. 2014;42(10):2363-70.

12. Sanders TL, Maradit Kremers H, Bryan AJ, Larson DR, Dahm DL, Levy BA, et al. Incidence of anterior cruciate ligament tears and reconstruction: a 21-year populationbased study. Am J Sports Med. 2016;44(6):1502-7.

13. Lind $M$, Menhert $F$, Pedersen AB. The first results from the Danish ACL reconstruction registry: epidemiologic and 2 year follow-up results from 5,818 knee ligament reconstructions. Knee Surg Sports Traumatol Arthrosc. 2009;17(2):117-24.

14. Buller LT, Best MJ, Baraga MG, Kaplan LD. Trends in anterior cruciate ligament reconstruction in the United States. Orthop J Sports Med.

2014;3(1):2325967114563664.

15. Maletis GB, Inacio MC, Funahashi TT. Analysis of 16,192 anterior cruciate ligament reconstructions from a community-based registry. Am J Sports Med. 2013;41(9):2090-8.

16. Kvist J, Kartus J, Karlsson J, Forssblad M. Results from the Swedish national anterior cruciate ligament register. Arthroscopy. 2014;30(7):803-10. Erratum in: Arthroscopy. 2014;30(8):1044.

17. Nordenvall R, Marcano AI, Adami J, Palme M, Mattila VM, Bahmanyar S, et al. The effect of socioeconomic status on the choice of treatment for patients with cruciate ligament injuries in the knee: a population-based cohort study. Am J Sports Med. 2017;45(3):535-40.

18. Granan LP, Forssblad M, Lind M, Engebretsen L. The Scandinavian ACL registries 2004-2007: baseline epidemiology. Acta Orthop. 2009;80(5):563-7.

19. Ahldén M, Samuelsson K, Sernert N, Forssblad M, Karlsson J, Kartus J. The Swedish National Anterior Cruciate Ligament Register: a report on baseline variables and outcomes of surgery for almost 18,000 patients. Am J Sports Med. 2012;40(10):2230-5.

20. Gliklich R, Dreyer N, Leavy M. Registries for Evaluating Patient Outcomes : A User ' s Guide. 3rd ed. Vol. 2. Rockville (MD): Agency for Healthcare Research and Quality (US); 2014.

21. Havelin LI, Engesaeter LB, Espehaug B, Furnes O, Lie SA, Vollset SE. The Norwegian Arthroplasty Register: 11 years and 73,000 arthroplasties. Acta Orthop Scand. 2000;71(4):337-53. 
22. Engebretsen $L$, Forssblad $M$, Lind $M$. Why registries analysing cruciate ligament surgery are important. Br J Sports Med. 2015;49(10):636-8.

23. Magnussen RA, Granan LP, Dunn WR, Amendola A, Andrish JT, Brophy R, et al. Cross-cultural comparison of patients undergoing ACL reconstruction in the United States and Norway. Knee Surg Sports Traumatol Arthrosc. 2010;18(1):98-105.

24. Maletis GB, Granan LP, Inacio MC, Funahashi TT, Engebretsen L. Comparison of community-based ACL reconstruction registries in the U.S. and Norway. J Bone Joint Surg Am. 2011;93 Suppl 3:31-6.

25. Mei Y, Ao YF, Wang JQ, Ma Y, Zhang X, Wang JN, et al. Clinical characteristics of 4355 patients with anterior cruciate ligament injury. Chin Med J (Engl). 2013;126(23):4487-92.

26. Renstrom P, Ljungqvist A, Arendt E, Beynnon B, Fukubayashi T, Garrett W, et al. Non-contact $A C L$ injuries in female athletes: an International Olympic Committee current concepts statement. Br J Sports Med. 2008;42(6):394-412. Review.

27. Leathers MP, Merz A, Wong J, Scott T, Wang JC, Hame SL. Trends and demographics in anterior cruciate ligament reconstruction in the United States. J Knee Surg. 2015;28(5):390-4.

28. Ventura A, Legnani C, Terzaghi C, Borgo E. Single- and double-bundle anterior cruciate ligament reconstruction in patients aged over 50 years. Arthroscopy. 2012;28(11):1702-9.

29. Desai N, Björnsson H, Samuelsson K, Karlsson J, Forssblad M. Outcomes after ACL reconstruction with focus on older patients: results from The Swedish National Anterior Cruciate Ligament Register. Knee Surg Sports Traumatol Arthrosc. 2014;22(2):379-86.

30. Tibor L, Chan PH, Funahashi TT, Wyatt R, Maletis GB, Inacio MC. Surgical technique trends in primary ACL reconstruction from 2007 to 2014. J Bone Joint Surg Am. 2016;98(13):1079-89.

31. Vascellari A, Grassi A, Combi A, Tomaello L, Canata GL, Zaffagnini S; SIGASCOT Sports Committee. Web-based survey results: surgeon practice patterns in Italy regarding anterior cruciate ligament reconstruction and rehabilitation. Knee Surg Sports Traumatol Arthrosc. 2017;25(8):2520-7.

32. Beynnon BD, Vacek PM, Newell MK, Tourville TW, Smith HC, Shultz SJ, et al. The effects of level of competition, sport, and sex on the incidence of first-time noncontact anterior cruciate ligament injury. Am J Sports Med. 2014;42(8):1806-12. 
33. Maletis GB, Inacio MC, Funahashi TT. Risk factors associated with revision and contralateral anterior cruciate ligament reconstructions in the Kaiser Permanente ACLR registry. Am J Sports Med. 2015;43(3):641-7.

34. Seng K, Appleby D, Lubowitz JH. Operative versus nonoperative treatment of anterior cruciate ligament rupture in patients aged 40 years or older: an expected-value decision analysis. Arthroscopy. 2008;24(8):914-20. Review. Erratum in: Arthroscopy. 2008;24(10):A14 .

35. Scillia AJ, Issa K, Boylan MR, McDermott JD, McInerney VK, Patel DV, et al. Inpatient cruciate ligament reconstruction in the United States: a nationwide database study from 1998 to 2010. Orthopedics. 2016;39(1):e196-202.

36. Lunebourg A, Ollivier M, Delahaye D, Argenson JA, Parratte S. Better satisfaction of patients operated on anterior cruciate ligament reconstruction in outpatient setting. $A$ prospective comparative monocentric study of 60 cases. Arch Orthop Trauma Surg. 2016;136(12):1709-15.

37. Mull HJ, Rivard PE, Legler A, Pizer SD, Hawn MT, Itani KMF, et al. Comparing definitions of outpatient surgery: implications for quality measurement. Am J Surg. 2017;214(2):186-92.

38. Cullen KA, Hall MJ, Golosinskiy A. Ambulatory surgery in the United States, 2006. Natl Health Stat Report. 2009;(11):1-25.

39. Duquin TR, Wind WM, Fineberg MS, Smolinski RJ, Buyea CM. Current trends in anterior cruciate ligament reconstruction. J Knee Surg. 2009;22(1):7-12.

40. Budny J, Fox J, Rauh M, Fineberg M. Emerging trends in anterior cruciate ligament reconstruction. J Knee Surg. 2017;30(1):63-9.

41. Lopes TJA, Simic M, Pappas E. Epidemiology of anterior cruciate ligament reconstruction in Brazil's public health system. Rev Bras Med Esporte. 2016;22(4):297301.

42. Middleton KK, Hamilton T, Irrgang JJ, Karlsson J, Harner CD, Fu FH. Anatomic anterior cruciate ligament $(\mathrm{ACL})$ reconstruction: a global perspective. Part 1. Knee Surg Sports Traumatol Arthrosc. 2014;22(7):1467-82.

43. Xie X, Liu X, Chen Z, Yu Y, Peng S, Li Q. A meta-analysis of bone-patellar tendonbone autograft versus four-strand hamstring tendon autograft for anterior cruciate ligament reconstruction. Knee. 2015;22(2):100-10. Review. 
44. Samuelsson K, Andersson D, Karlsson J. Treatment of anterior cruciate ligament injuries with special reference to graft type and surgical technique: an assessment of randomized controlled trials. Arthroscopy. 2009;25(10):1139-74. Review.

45. DeAngelis JP, Fulkerson JP. Quadriceps tendon--a reliable alternative for reconstruction of the anterior cruciate ligament. Clin Sports Med. 2007;26(4):587-96. Review.

46. Mulford JS, Hutchinson SE, Hang JR. Outcomes for primary anterior cruciate reconstruction with the quadriceps autograft: a systematic review. Knee Surg Sports Traumatol Arthrosc. 2013;21(8):1882-8. Review.

47. Maletis GB, Inacio MC, Desmond JL, Funahashi TT. Reconstruction of the anterior cruciate ligament: association of graft choice with increased risk of early revision. Bone Joint J. 2013;95-B(5):623-8.

48. Hagino T, Ochiai S, Senga S, Yamashita T, Wako M, Ando T, et al. Meniscal tears associated with anterior cruciate ligament injury. Arch Orthop Trauma Surg.

2015;135(12):1701-6.

49. Øiestad BE, Engebretsen L, Storheim K, Risberg MA. Knee osteoarthritis after anterior cruciate ligament injury: a systematic review. Am J Sports Med.

2009;37(7):1434-43. Review.

50. Brambilla L, Pulici L, Carimati G, Quaglia A, Prospero E, Bait C, et al. Prevalence of associated lesions in anterior cruciate ligament reconstruction: correlation with surgical timing and with patient age, sex, and body mass index. Am J Sports Med. 2015;43(12):2966-73.

51. Ageberg E, Forssblad M, Herbertsson P, Roos EM. Sex differences in patient-reported outcomes after anterior cruciate ligament reconstruction: data from the Swedish knee ligament register. Am J Sports Med. 2010;38(7):1334-42.

52. Stein T, Mehling AP, Welsch F, von Eisenhart-Rothe R, Jäger A. Long-term outcome after arthroscopic meniscal repair versus arthroscopic partial meniscectomy for traumatic meniscal tears. Am J Sports Med. 2010;38(8):1542-8.

53. Paxton ES, Stock MV, Brophy RH. Meniscal repair versus partial meniscectomy: a systematic review comparing reoperation rates and clinical outcomes. Arthroscopy. 2011;27(9):1275-88. Review.

54. Granan LP, Inacio MC, Maletis GB, Funahashi TT, Engebretsen L. Intraoperative findings and procedures in culturally and geographically different patient and surgeon populations: an anterior cruciate ligament reconstruction registry comparison between Norway and the USA. Acta Orthop. 2012;83(6):577-82. 
55. Fetzer GB, Spindler KP, Amendola A, Andrish JT, Bergfeld JA, Dunn WR, et al. Potential market for new meniscus repair strategies: evaluation of the MOON cohort. J Knee Surg. 2009;22(3):180-6.

56. Noyes FR, Barber-Westin SD. Treatment of meniscus tears during anterior cruciate ligament reconstruction. Arthroscopy. 2012;28(1):123-30. Review.

57. Parker BR, Hurwitz S, Spang J, Creighton R, Kamath G. Surgical trends in the treatment of meniscal tears: analysis of data from the American Board of Orthopaedic Surgery Certification Examination Database. Am J Sports Med. 2016;44(7):1717-23.

58. Abrams GD, Frank RM, Gupta AK, Harris JD, McCormick FM, Cole BJ. Trends in meniscus repair and meniscectomy in the United States, 2005-2011. Am J Sports Med. 2013;41(10):2333-9.

59. Astur DC, Batista RF, Gustavo A, Cohen M. Trends in treatment of anterior cruciate ligament injuries of the knee in the public and private healthcare systems of Brazil. Sao Paulo Med J. 2013;131(4):257-63.

60. Yoon KH, Park KH. Meniscal repair. Knee Surg Relat Res. 2014;26(2):68-76. Review.

61. Westermann RW, Lynch TS, Spindler KP. Hot topics in the Multicenter Orthopedics Outcomes Network: anterior cruciate ligament. J Knee Surg. 2016;29(7):539-42. Review.

62. Coşkunsu D, Bayrakcı Tunay V, Akgün I. Current trends in reconstruction surgery and rehabilitation of anterior cruciate ligament in Turkey. Acta Orthop Traumatol Turc. 2010;44(6):458-63.

63. Martimbianco AL, Gomes da Silva BN, de Carvalho AP, Silva V, Torloni MR, Peccin MS. Effectiveness and safety of cryotherapy after arthroscopic anterior cruciate ligament reconstruction. A systematic review of the literature. Phys Ther Sport. 2014;15(4):261-8. Review.

64. Wright RW, Haas AK, Anderson J, Calabrese G, Cavanaugh J, Hewett TE, Lorring D, McKenzie C, Preston E, Williams G; MOON Group. Anterior cruciate ligament reconstruction rehabilitation: MOON Guidelines. Sports Health. 2015;7(3):239-43.

65. Dhawan A, Doukas WC, Papazis JA, Scoville CR. Effect of drain use in the early postoperative period after arthroscopically assisted anterior cruciate ligament reconstruction with bone-patellar tendon-bone graft. Am J Sports Med. 2003;31(3):419-24. 
66. McCormack RG, Greenhow RJ, Fogagnolo F, Shrier I. Intra-articular drain versus no drain after arthroscopic anterior cruciate ligament reconstruction: a randomized, prospective clinical trial. Arthroscopy. 2006;22(8):889-93.

67. Straw R, Colclough K, Geutjens GG. Arthroscopically assisted ACL reconstruction. Is a drain necessary? Knee. 2003;10(3):283-5.

68. Bryson DJ, Morris DL, Shivji FS, Rollins KR, Snape S, Ollivere BJ. Antibiotic prophylaxis in orthopaedic surgery: difficult decisions in an era of evolving antibiotic resistance. Bone Joint J. 2016;98-B(8):1014-9. Review.

69. Prokuski L. Prophylactic antibiotics in orthopaedic surgery. J Am Acad Orthop Surg. 2008;16(5):283-93. Review.

70. Keller RA, Moutzouros V, Dines JS, Bush-Joseph CA, Limpisvasti O. Deep venous thrombosis prophylaxis in anterior cruciate ligament reconstructive surgery: what is the current state of practice? Sports Health. 2018;10(2):156-9.

71. Werner BC, Burrus MT, Kew ME, Dempsey IJ, Gwathmey FW, Miller MD, et al. Limited utility of routine early postoperative radiography after primary $A C L$ reconstruction. Knee. 2016;23(2):237-40.

72. Stucken C, Flato R, O'Hagan T, O'Brien D, Ruiz D, Cohen SB, et al. Postoperative radiographs after $A C L$ reconstruction are not cost-effective. Orthopedics.

2015;38(4):e339-42. 


\begin{abstract}
Introduction: Anterior cruciate ligament rupture is a common athletic injury and has been reported as the knee ligament injury that most often requires surgical reconstruction. Purpose: To describe the epidemiological characteristics of the anterior cruciate ligament reconstruction and determine trends in medical practice. Methods: We retrospectively reviewed the anterior cruciate ligament institutional register to obtain data from all patients who underwent primary anterior cruciate ligament reconstruction from July 2014 to June 2016. Patients who underwent either revision or other concomitant knee ligaments surgeries were excluded. Descriptive statistics were used to summarize the sample. It was also assessed association between meniscal lesion and other variables. Results: During the study period, 500 patients were included. $72.6 \%$ of patients were male. Mean age at surgery was 35.1 years. Mean age among females was higher than among males (37.3 \pm 12.1 versus $34.3 \pm 10.8)$. The most common femoral and tibial fixation used was suspensory fixation (60.8\%) and interference screw (96\%), respectively. The most commonly used graft was hamstrings tendons $(70.2 \%)$ followed by bone-patellar tendon-bone (28.8\%). Allograft was used in only one case $(0.2 \%)$. Meniscal lesion was noted in $44.8 \%$ of cases. Medial meniscus was the most injured (59.4\%) and longitudinal lesions were the most frequent $(50.6 \%)$. Partial meniscectomy was performed in $69.5 \%$ of meniscal lesions and meniscal repair in $14.4 \%$. The mean length of hospital stay was 1.4 days. In $25.4 \%$ of patients, a drain was used after surgery. There is no readmission during the study period. The proportion of men in the group of patients with associated meniscal lesion was higher than in the group of patients without meniscal lesion $(p=0.007)$. There was no association between meniscal lesion and the following variables: age, body mass index and time from injury to surgery. Conclusions: The majority of surgeries were performed in male patients in all age groups, with predominance in patients older than 30 years and short time period from injury to surgery. Concerning trends in surgical technique, there was low rate of meniscal repair and higher preference for the use of the hamstring tendon graft and suspensory fixation on the femoral side.
\end{abstract}

